\title{
Frequency Split Analysis of a Ring Vibratory Gyroscope Based on Harmonic Transformation of Parametric Errors
}

Xiang Xi ( $\boldsymbol{\nabla}$ xixiang@nudt.edu.cn )

national university of defense technology

Yongmeng Zhang

National university of defense technology

Jiangkun Sun

National university of defense technology

Xuezhong Wu

National university of defense technology

Original Article

Keywords: Ring vibratory gyroscope, Frequency split, Harmonic transformation, Parametric errors

Posted Date: August 26th, 2020

DOI: https://doi.org/10.21203/rs.3.rs-63047/v1

License: @ (i) This work is licensed under a Creative Commons Attribution 4.0 International License.

Read Full License 


\section{Title page}

\section{Frequency Split Analysis of a Ring Vibratory Gyroscope Based on Harmonic Transformation of Parametric Errors}

Xiang Xi, born in 1986, is currently an associate professor at College of Intelligence Science, National University of Defense Technology, China. He received his $\mathrm{PhD}$ degree from National University of Defense Technology, China, in 2014. His research interests include inertial sensors, micro-electromechanical systems and mechanical engineering.

Tel: +86-0731-84574958; E-mail: xixiang@ nudt.deu.cn

Yong-Meng Zhang, born in 1988, is currently a lecturer at College of Intelligence Science, National University of Defense Technology, China. He received his $\mathrm{PhD}$ degree from National University of Defense Technology, China, in 2017. His research interests include inertial sensors, micro-electromechanical systems and control of gyroscope.

Tel: +86-0731-84574958; E-mail: zhangym@nudt.edu.cn

Jiang-Kun Sun, born in 1992, is currently a $\mathrm{PhD}$ candidate at College of Intelligence Science, National University of Defense Technology, China.

E-mail: sunjiangkun15@nudt.edu.cn

Xue-Zhong Wu, born in 1965, is currently a professor at National University of Defense Technology, China. He received his PhD degree from National University of Defense Technology, China, in 1988. His research interests include inertial sensors, micro-electromechanical systems and precise manufacture.

Tel: +86-0731-84574958; E-mail: xzwu@ nudt.edu.cn

\section{Corresponding author: Xiang Xi E-mail: xixiang@nudt.edu.cn}




\title{
Frequency Split Analysis of a Ring Vibratory Gyroscope Based on Harmonic Transformation of Parametric Errors
}

\author{
Xiang Xi • Yong-Meng Zhang • Jiang-Kun Sun $・$ Xue-Zhong Wu
}

Received June xx, 201x; revised February xx, 201x; accepted March xx, 201x

(C) Chinese Mechanical Engineering Society and Springer-Verlag Berlin Heidelberg 2017

\begin{abstract}
'Abstract: Ring vibratory gyroscopes are important angular rate sensors widely used in inertial navigation systems. A highly symmetrical resonator is the core part of the ring vibratory gyroscope. Frequency split is a key parameter which denotes the level of unbalanced mass and stiffness of the resonator. Many research works focus on the precise machining of the resonator for the sake of eliminating frequency split. However, for metallic ring resonators, the decrease of frequency split is not always proportional to the promotion of geometric accuracy. This paper investigates the frequency split of the ring resonator gyroscope caused by parametric errors including geometric and material imperfection via a method of harmonic transformation. The roundness error of the ring resonator is extracted, and then decomposed to a series of orders of harmonic waves. Transformation results show that for the tested resonator, its first 20 orders of harmonic waves contain the main components of the roundness error. Then a precise FEM modeling is built for frequency split analysis. The simulation result shows that the roundness error of the resonator can cause a frequency split of $0.6 \mathrm{~Hz}$, which accounts for $16.2 \%$ of the total frequency split. Based on the metallographic observation and grouping experiment of different metallic resonators, it is deduced that the main frequency split is caused by material heterogeneity. It shows that the material homogenization is as important as precise machining for the resonator of small frequency split. The proposed research provides an instruction to manufacture high quality metallic resonators.
\end{abstract}

Keywords: Ring vibratory gyroscope - Frequency split • Harmonic transformation $\bullet$ Parametric errors

Xiang Xi

xixiang@nudt.edu.cn

College of Intelligence Science, National University of Defense Technology, Changsha 410073, China

\section{Introduction}

A ring vibratory gyroscope is a type of angular rate sensor which can be widely used in the field of inertial navigation, attitude measurement, and robotic sensing, etc [1-3]. It is attracting increasingly attention in recent years because of merits of high precision, low cost and long life. An ideal balanced resonator is necessary for mode-matching of the ring vibratory gyroscope, and thus makes the gyroscope have a high sensitivity and small zero bias [4,5]. The frequency split, denoting the level of unbalanced mass and stiffness of the resonator, is a key parameter relating to the performance of the gyroscope [6,7]. The rising of the frequency split, widely known as parametric errors such as geometric imperfection and material heterogeneity, has been studied by many researchers [8,9]. Most frequency split analyses are based on the model of fused silica hemispherical resonator gyroscope (HRG) by employing a hypothesis of density or radius variation. Or the imperfection is equivalent to point masses. Choi et al. [7] estimated the influences of point masses on frequency split and mode shift of an imperfect hemispherical shell. Bisegna et al. [10] modeled the imperfections as perturbations of the uniform linear mass density and bending stiffness on a perfect ring. However, actual geometric topography of the resonator is irregular and complicated. Usually the roundness error of the resonator is only several micro meters or even sub-micron. Therefore the prediction of the frequency split before manufacture of the resonator would be very difficult. Senkal et al. [11] demonstrated a micro resonator of sub- $1 \mathrm{~Hz}$ frequency split, and analyzed its frequency split caused by thickness error. Weinberg [12] studied the frequency split caused by the $2 n$ harmonic of density or thickness variation by using 
FEM simulation. But most previous work did not give a direct transformation between the frequency split and the parametric errors of the resonator.

Our previous work studied some metallic vibratory ring gyroscopes which have advantage of low cost and easy maufacturing [13-16]. However, the frequency split of the metallic resonator is relative large because the material uniformity of the metal is not as good as that of fused silica. Yang et al. [17] proposed a balancing method of a metallic cylindrical resonator with a frequency split of $12.4 \mathrm{~Hz}$. Lin et al. [18] studied the trimming method of a metallic resonator with stochastic imperfections by adding an imperfect mass point to a perfect shell. $\mathrm{Hu}$ et al. [19] studied the influence of mass and stiffness imperfection on modal axis of the resonator, which provides a guarantee for high efficiency and high precision trimming. During the frequency split analysis, the influence of the geometric error is difficult to be isolated since it is always affecting the resonant frequencies in combination with material inhomogeneity. Therefore in the resonator manufacture, an anomaly is that the value of frequency split is not proportional to the geometric accuracy of the resonator. In order to build a controllable machining process, it is meaningful to analysis the influence of each parametric error.

Harmonic transformation is a novel method to identify the characteristics of mechanical elements. Wei et al. [20] proposed a separation method of roundness error based on single transposition characteristic angle, which improves the measurement accuracy of the shape errors for a standard optical ball. Raine et al. [21] analyzed the phase and amplitude of harmonic components of a bearing inner ring, which is considered helpful for improving the manufacturing accuracy. Augustin et al. [22] studied the application of Fourier series for evaluation of roundness profiles in metrology.

Inspired by the method of harmonic transformation applied in evaluation of the roundness error, this paper studies the frequency split of a ring vibratory gyroscope based on harmonic transformation of the parametric errors. Basic concepts are introduced in Section 2, and the harmonic transformation method in combination with accurate FEM modeling is proposed to obtain numerical results of frequency split in Section 3. Then the roundness error of a resonator sample is experimentally measured in Section 4. Its thickness error in form of harmonic waves is extracted by using the harmonic transformation. Finally,

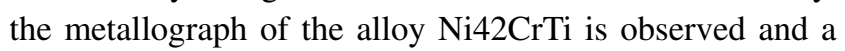
grouping experiment is designed to explore the influence of the inhomogeneous material.

\section{Frequency Split of the Ring Resonator}

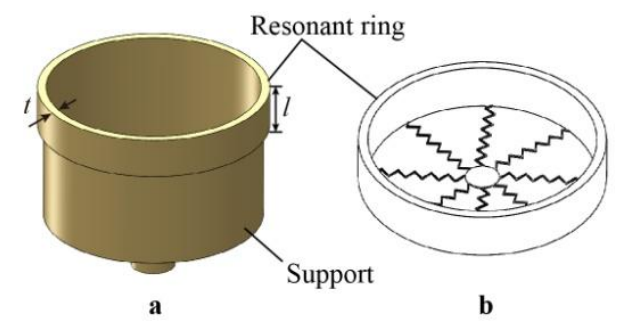

Figure 1 The metallic resonator and its ring model, a resonator, b ring model

Table 1 Main sizes of the ring resonator

\begin{tabular}{lccc}
\hline Parameter & $\begin{array}{c}\text { Radius } \\
r /(\mathrm{mm})\end{array}$ & $\begin{array}{c}\text { Ring thickness } \\
t /(\mathrm{mm})\end{array}$ & $\begin{array}{c}\text { Ring length } \\
l /(\mathrm{mm})\end{array}$ \\
\hline Size & 12.5 & 1 & 5 \\
\hline
\end{tabular}

Figure 1(a) shows the resonator structure of the studied ring vibratory gyroscope. Its core part is a resonant ring supported by a thin cylinder. Therefore in theory it can be equivalent to a short ring with elastic support of springs, as shown in Figure 1(b). Typical sizes of the resonator are listed in Table 1. Different with MEMS ring vibratory gyroscopes or fused silica hemispherical resonator gyroscope, the metallic ring resonator can be fabricated via simple mechanical processing like turning or grinding. Benefit from modern alloy material, high elastic alloy such as Ni42CrTi (3J53) shows good frequency temperature coefficient even better than fused silica, which is suitable for the manufacture of tactical gyroscopes with low cost.

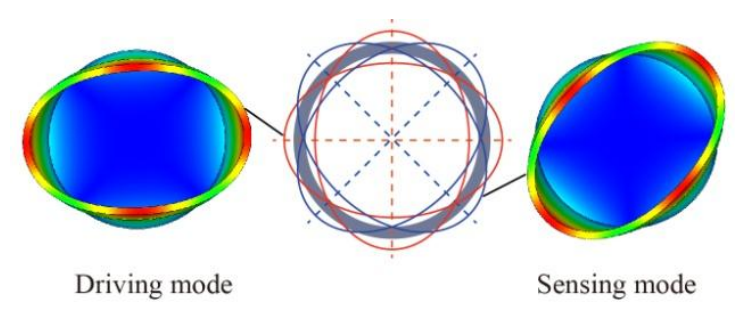

Figure 2 Operating mode of the ring resonator

The operating of the ring vibratory gyroscope relies on the standing wave vibration of the resonator. Under the excitation of a sinusoidal force, the resonator vibrates in the form of elliptical driving mode. When a rotation is input, the sensing mode is excited due to the force induced by Coriolis effect, as shown in Figure 2. Therefore it is important to make the two frequencies match with each other, according to the scale factor (SF) formula of the symmetric resonant structure [23]: 


$$
\mathrm{SF} \propto \sqrt{\frac{1}{\tau_{d}^{2}}+\left(\omega_{d}-\omega_{f}\right)^{2}} \cdot \sqrt{\frac{1}{\tau_{s}^{2}}+\left(\omega_{s}-\omega_{f}\right)^{2}},
$$

where $\omega_{d}$ and $\omega_{s}$ are, respectively, the eigenfrequencies of the driving and sensing modes, and $\omega_{f}$ is the exciting frequency. $\tau_{d}$ and $\tau_{s}$ are the damping time constants of the resonator. It can be seen that when $\omega_{d}=\omega_{s}=\omega_{f}$, the scale factor reaches the biggest value, meaning that the gyroscope has a high sensitivity. Moreover, the frequency split causes drift of the standing wave when the resonator vibratory in a free processing mode [24]:

$$
\dot{\theta}=k\left(\omega_{d}-\omega_{f}\right)^{2} f(\varphi),
$$

where $k$ is a constant coefficient, and $f(\varphi)$ is a sine function related to the angle of the standing wave vibration.

Due to the geometric errors and material imperfection of the resonator, there is always a frequency split between the driving eigenfrequency $\omega_{d}$ and the sensing eigenfrequency $\omega_{s}$. Usually the geometric error of the resonator can be evaluated by parameters such as roundness and concentricity. However, they cannot be used to evaluate the frequency split of the resonator because a simple roundness or concentricity is not sufficient to express the accurate geometrical morphology.

\section{Harmonic Transformation of Parametric Errors}

In order to analysis the relation between the parametric errors and the frequency split of the ring resonator, a method of harmonic transformation is introduced in this section.

\subsection{Theoretical Consideration}

Based on Fourier theory, the real parameter distribution of the resonant ring $p(\theta)$ can be written as combination of harmonic functions:

$$
\begin{aligned}
& p(\theta)=p_{0}+\sum_{n=1}^{\infty}\left(a_{n} \cos n \theta+b_{n} \sin n \theta\right), \\
& =p_{0}+\sum_{n=1}^{\infty} c_{n} \cos \left(n \theta-\alpha_{n}\right)
\end{aligned},
$$

where $p_{0}$ is the average parameter such as average radius, thickness or density. $\theta$ is the angular variation, $n$ is the nature number which denotes the order of the harmonic wave, and $a_{n}, b_{n}$ are the coefficients related to the error distribution:

$$
\left\{\begin{array}{l}
a_{n}=\frac{1}{\pi} \int_{0}^{2 \pi} p \cos n \theta \mathrm{d} \theta \\
b_{n}=\frac{1}{\pi} \int_{0}^{2 \pi} p \sin n \theta \mathrm{d} \theta, \\
r_{0}=\frac{1}{\pi} \int_{0}^{2 \pi} p \mathrm{~d} \theta
\end{array}\right.
$$

The second line of Eq. (3) is another form of the transformation including a phase parameter. If an eccentric error with coordinate of $e_{x}$ and $e_{y}$ is introduced by the measurement system, Eq. (3) can be rewritten as:

$$
\begin{aligned}
& p(\theta)=\left\{\left\{\left[p_{0}+\sum_{n=1}^{\infty}\left(a_{n} \cos n \theta+b_{n} \sin n \theta\right)\right] \cos \theta+e_{x}\right\}^{2}\right. \\
& \left.+\left\{\left[p_{0}+\sum_{n=1}^{\infty}\left(a_{n} \cos n \theta+b_{n} \sin n \theta\right)\right] \sin \theta+e_{y}\right\}^{2}\right\}^{1 / 2}
\end{aligned}
$$

Therefore the parametric error of the resonant ring is transformed to a series of harmonic functions. This transformation can decompose the irregular parameter into sine and cosine function. Hence the influence of the parametric errors on the frequency split can be separated. Detailed error forms of the resonator can be figured out based on Eq. (3) and as shown in Figure 3 (only first 4 orders of harmonic errors are listed). Three types of parametric errors are listed in the picture. Type $I$ is the form of the shape error. In this case, the thickness of the resonator is homogeneous, but the deformations are different. Type II is the form of the thickness errors. Type III denotes parametric errors of the material such as variation of density and elastic modulus (blue area represents a parameter smaller than average and black area represents a parameter larger than average).

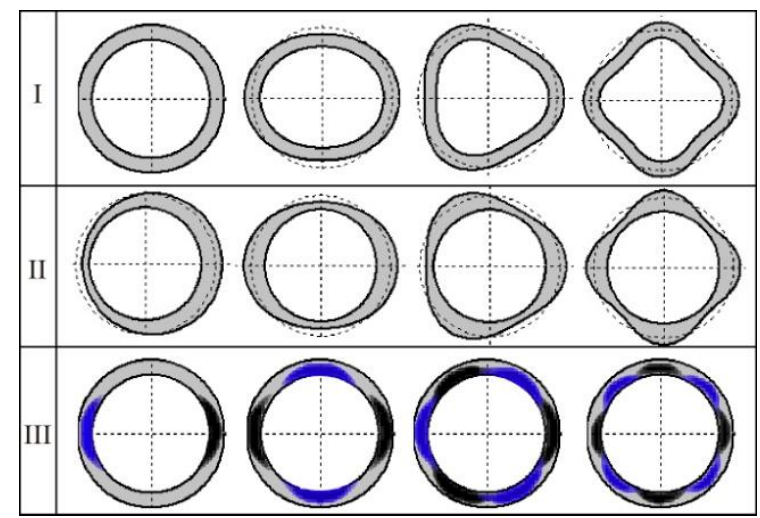

Figure 3 Decomposition of the geometric errors of the ring resonators 
It can be seen that for different types of parametric errors, the mass and stiffness distribution of the resonator is periodic. The dynamic equation of an imperfect ring resonator under parametric disturbance can be expressed as [10]

$$
\begin{aligned}
& -\left(\omega_{0}+\delta \omega\right)^{2} \int_{0}^{2 \pi}\left(m_{0}+\delta m\right)\left[\left(u_{0}+\delta u\right) \Psi+\frac{\partial\left(u_{0}+\delta u\right)}{\partial \theta} \frac{\partial \Psi}{\partial \theta}\right] R d \theta \\
& +\int_{0}^{2 \pi} \frac{\left(K_{0}+\delta K\right)}{R^{4}}\left(\frac{\partial}{\partial \theta}+\frac{\partial^{3}}{\partial \theta^{3}}\right)\left(u_{0}+\delta u\right)\left(\frac{\partial \Psi}{\partial \theta}+\frac{\partial^{3} \Psi}{\partial \theta^{3}}\right) R d \theta=0
\end{aligned}
$$

where $\delta \rho$ and $\delta K$ are, respectively, $2 \pi$-periodic perturbations of the mass density $m_{0}$ and the bending stiffness $K_{0}$ of the perfect ring resonator. The corresponding vibration amplitude on eigenfrequency $\omega_{0}$ of the perfect resonator is represented as $u_{0} . \Psi$ is the test function for Fourier series expansions.

Eq. (6) reveals a relation between the harmonic transformation of parametric errors and the frequency variation of the resonator. Considering that the theoretical analytical solution is too complicated for all the parametric errors, a FEM simulation is introduced in the following section.

\subsection{Simulation}

In this simulation, the influences of different types of parametric errors on frequency split are studied. The geometric parameters of the resonator used for simulation are listed in Table 1. The density of the alloy material is $7850 \mathrm{~kg} / \mathrm{m}^{3}$. Its Poisson's ratio is 0.28 and the Young's modulus is $210 \mathrm{GPa}$. FEM software ANSYS is used for model building and solution. Without loss of generality, the relative error in the model is set within $1 \%$ of the original parameter.

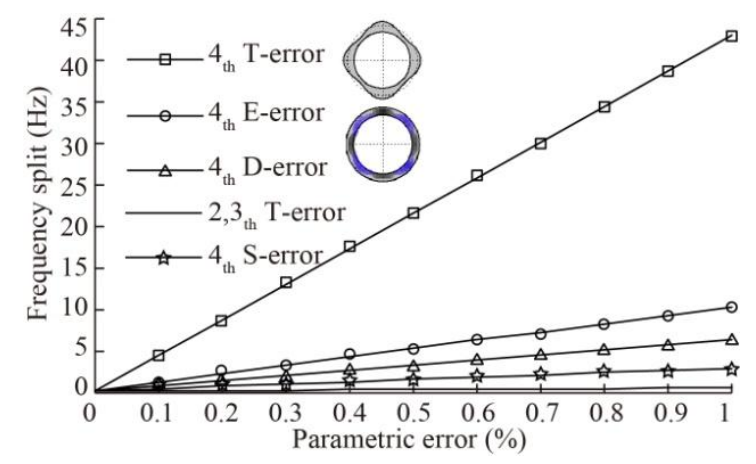

Figure 4 Frequency split dependent on harmonic wave order

Simulation results of parametric errors in form of different harmonic waves are shown in Figure 4. It can be seen that, overall, the frequency split generated by the $4^{\text {th }}$ harmonic error is much bigger than that generated by other harmonic errors. $1 \%$ of $4^{\text {th }}$ thickness harmonic error (T-error in the picture) can cause a frequency split of 41.6 $\mathrm{Hz}$, which is almost unacceptable for use of the gyroscope. This phenomenon can be explained as that the elliptical standing wave mode is sensitive to the $4^{\text {th }}$ harmonic error. In this error form, the resonator has lumped mass and stiffness along one of its modal axes. While along the other matched modal axis, spaced $45^{\circ}$ with each other, the local mass and stiffness is the smallest. It is noted that as the increase of the ring thickness, the according mass and thickness increase at the same time. But based on the classic frequency calculation formula $\omega=(K / m)^{1 / 2}$, they influence frequency in a reverse way. Considering that the stiffness variation is the cubic function of the thickness variation, while the mass variation is the linear function of the thickness variation. Therefore, the frequency split caused by $4^{\text {th }}$ thickness harmonic error is actually dominated by stiffness variation changed with ring thickness.

$4^{\text {th }}$ elastic harmonic error (E-error), density harmonic error (D-error) and shape harmonic error (S-error) also contribute to the rise of frequency split. $2,3^{\text {th }}$ thickness harmonic error generates a frequency split less than $0.5 \mathrm{~Hz}$. Other orders of harmonic errors (larger than $4^{\text {th }}$ ) are not shown in the picture because they generate little frequency split compared with the $4^{\text {th }}$ harmonic error.

\section{Geometric Error and Frequency Split of the Ring Resonator}

In order to predict the frequency split caused by geometric error, a real ring resonator is measured and the harmonic transformation is implemented. Then a precise finite element model is built according to the measured roundness of the resonator. Therefore the frequency split of the resonator is caused by geometric error is separated.

\subsection{Harmonic Error Analysis}

Figure 5 shows the roundness error of the ring resonator sample, measured by a roundness instrument (RA160, CHINATEST). The roundness errors of resonator's inner circle and outer circle are $3.48 \mu \mathrm{m}$ and $6.07 \mu \mathrm{m}$ respectively, and the according thickness error is also indicated in Figure 5. In the measurement process, the starting points of inner circle and outer circle must be the same. Note that the geometric shape of the resonator is mainly affected by clamping deformation. Along different 
axial positions of the resonator, their outlines would have a small difference, but basically their geometric shapes are very similar. Therefore only one outline is measured since that the resonant ring is relatively short.

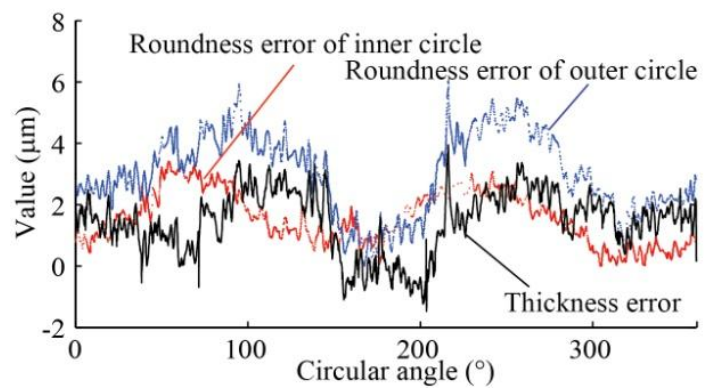

Figure 5 Roundness and thickness errors of the resonator.

In the study of Section 3, it is known that the frequency split is mainly affected by the $4^{\text {th }}$ thickness harmonic error. Then the harmonic transformation of the thickness error is implemented here. The first 100 orders of harmonic waves are fit in the transformation, as shown in Figure 6. In the picture, the amplitude of the transformation is the roundness error for each harmonic wave component. The phase information, denoting the orientation of the harmonic wave, can be also obtained from the transformation but not shown in the picture. It can be found that the first 20 orders of harmonic errors are significantly larger than the other orders of harmonic errors. Especially, the $2^{\text {nd }}$ and $3^{\text {rd }}$ harmonic errors are the biggest error components. It is deduced that the $2^{\text {nd }}$ harmonic error is caused by the bending deformation of the shell under the axial tension, and the $3^{\text {rd }}$ harmonic error is caused by clamping of the three jaw chuck during the manufacturing process.

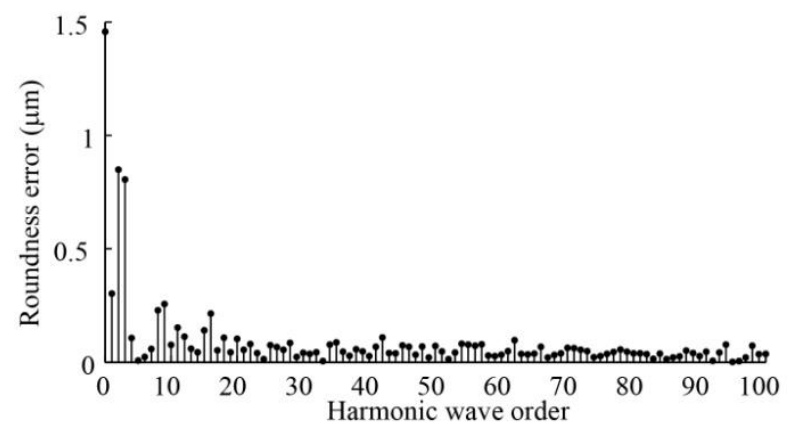

Figure 6 Roundness errors related to the orders of the harmonic waves

Figure 7 is the fitting curve of the transformation, obtained by the composition of the first 4 orders of the harmonic waves based on Eq. (3). It can be seen that the fitting outline of is very close to the actual roundness error of the resonator. If the fitting order is increased to 100 , the fitting outline is almost the same with the actual roundness outline, as shown in Figure 8. The fitting results show that for the manufacture of the metallic ring resonator, it is very important to limit the errors of the first 4 orders of the harmonic errors. Hence the traditional jigs with 3 or 4 chucks should be avoided to fix the resonator during the machining process.

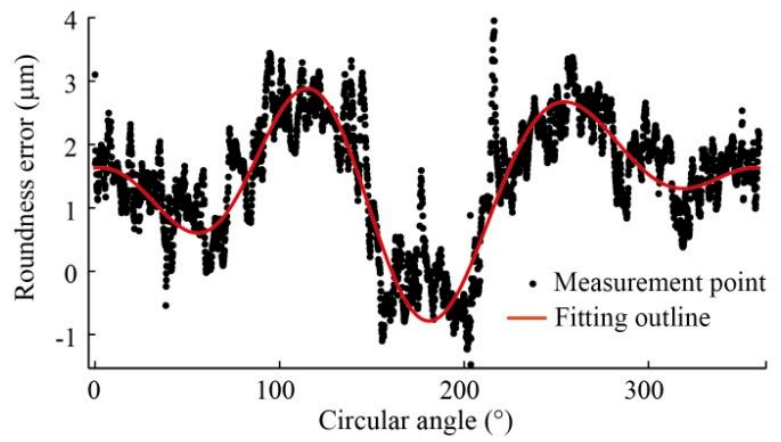

Figure 7 Fitting of roundness error using first 4 orders of harmonic waves

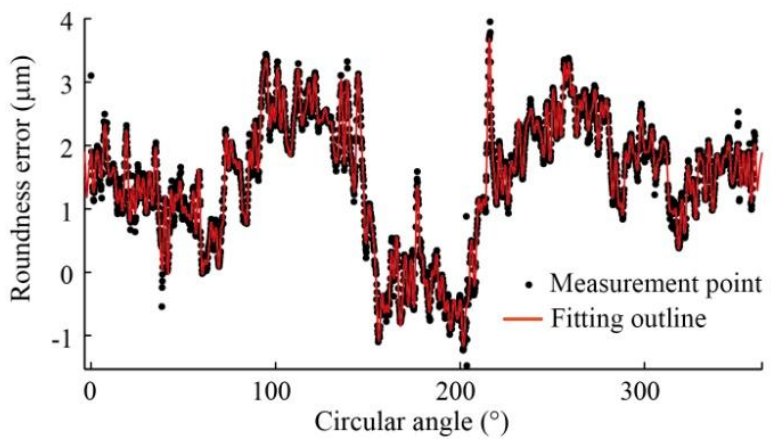

Figure 8 Fitting of roundness error using first 100 orders of harmonic waves

\subsection{FEM Modeling for Frequency Split Analysis}

A FEM model having the same geometric and material parameters with the actual sample in Section III is built by ANSYS software. The roundness error is included in the model to obtain its influence on the frequency split.

Main steps of the FEM modeling are listed as follows: (1) the measured outline of the resonator is introduced to CAD software to build a precise geometric model with thickness imperfection. (2) Then the imperfect model close to the actual sample is imported into the FEM software ANSYS. It should be noted that in order to make the FEM nodes accurately fit the outline of the resonator, the size of the element must be small enough. In this simulation, it is set as $100 \mu \mathrm{m}$. Figure 9 indicates the meshing accuracy of the FEM modeling for the frequency split analysis. It can be seen that after meshing the FEM nodes can be exactly 
overlapped with the measurement line. (3) The modal simulation is conducted to obtain the eigenfrequency of the ring resonator.

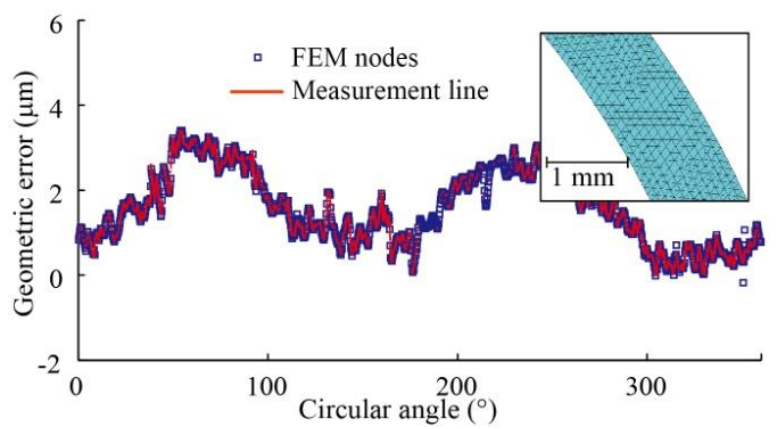

Figure 9 FEM modeling for frequency split analysis

The simulation result is shown in Figure 10. The two split eigenfrequencies of the ring resonator are $4034.4 \mathrm{~Hz}$ and $4035 \mathrm{~Hz}$ respectively, i.e. the frequency split is $0.6 \mathrm{~Hz}$. The eigenmode contour of the resonator is shown in Figure 10(a). It can be seen that there is a deflection of the modal axis. The deflection angle is $9.7^{\circ}$, which is equal to the phase angle of the $4^{\text {th }}$ thickness harmonic error. The amplitude of the $4^{\text {th }}$ thickness harmonic error is $0.107 \mu \mathrm{m}$, which can cause about $0.47 \mathrm{~Hz}$ frequency split in theory. The $2^{\text {nd }}$ and $3^{\text {rd }}$ harmonic errors also contribute to a small proportion of the total frequency split.

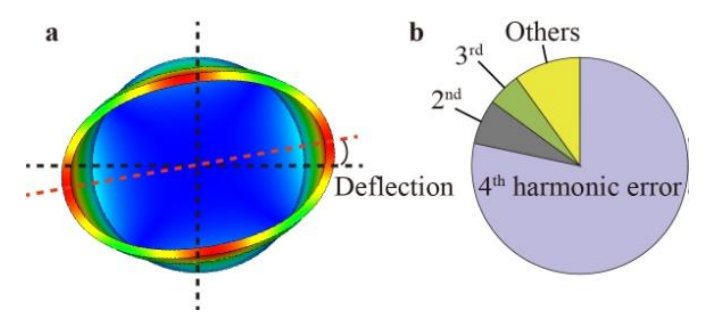

Figure 10 FEM result of the resonator, a eigenmode contour, $\mathbf{b}$ frequency split dependent on different harmonic errors

\section{Influence of Inhomogeneous Material}

From previous study the frequency split related to the geometric errors is obtained. It illustrates the frequency split of the resonator in a case of perfect material. However, it is well known that there is always imperfection such as density and elastic variation in the metallic alloy. Therefore, a frequency response of the tested resonator (the same resonator used for previous test) is further obtained. In the frequency response test, the resonator is excited by using an electromagnetic device and the vibration is detected by using a microphone. This non-contact modal testing method can obtain the accurate eigenfrequencies of the resonator. More details about the experimental setup can be found in reference [25].

The testing result is shown in Figure 11. It can be found that the measured eigenfrequencies of the resonator are $3935.7 \mathrm{~Hz}$ and $3939.4 \mathrm{~Hz}$, namely the frequency split is $3.7 \mathrm{~Hz}$, which is significantly larger than the FEM result of $0.6 \mathrm{~Hz}$. It means that for this resonator sample, the geometric error only causes a small proportion $(16.2 \%)$ of the total frequency split.

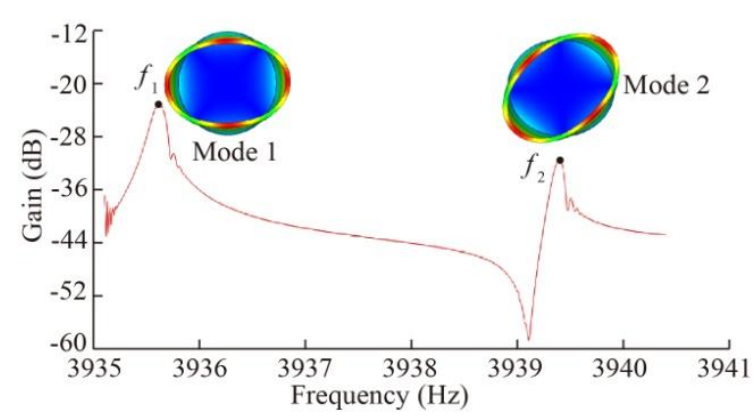

Figure 11 Frequency response of the imperfect ring resonator

From the theoretical analysis, it can be deduced that the other portion of the frequency split is caused by the material heterogeneity. Therefore, the metallographic pictures of the Ni42CrTi alloy are observed by using a microscope, as shown in Figure 12. The measured points are from two symmetric positions on the resonator rim. It can be seen that the grain size and precipitate phase from the two positions have significant differences. This is caused by the imperfection from the formation of the alloy and the heat treatment process.

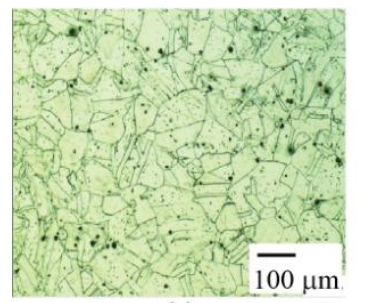

(a)

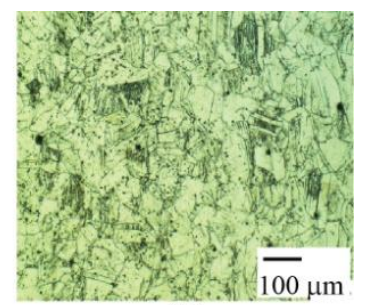

(b)
Figure 12 Metallograph from different positions of the alloy $\mathrm{Ni42CrTi}$

However, it is very hard to obtain the distribution of the density or stiffness of the whole resonator. Therefore a theoretical or FEM model is not available for analysis of frequency split caused by inhomogeneous material. A grouping experiment based on different alloys is designed. One group of the resonators is made of elastic copper, which has good material uniformity and machinability. 
The other group of the resonators is still made of $\mathrm{Ni} 42 \mathrm{CrTi}$, which has a better frequency temperature coefficient. In each group, 7 resonators were manufactured. In the manufacturing process, the technical requirement, machining parameters and the jig are tried to keep the same. Therefore the roundness of the resonators can be roughly controlled to be the same (between $1 \mu \mathrm{m}$ to $2 \mu \mathrm{m}$ ). The testing results of the frequency split are shown in Figure 13. It can be observed that the frequency splits of most copper resonators are smaller than those of $\mathrm{Ni} 42 \mathrm{CrTi}$ resonators. This is because that the elastic copper material has a single metallic element, which is critical for the material uniformity.

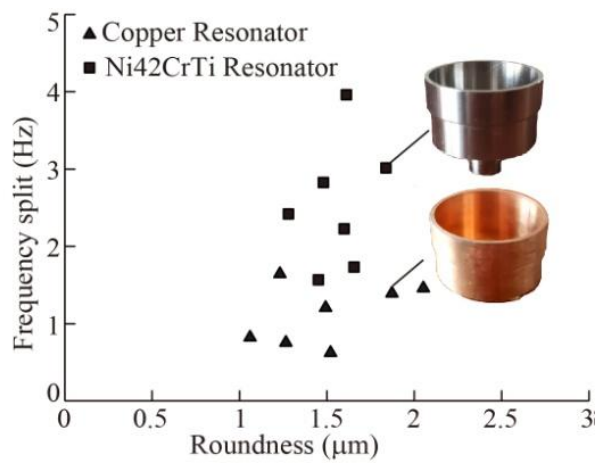

Figure 13 Metallograph from different positions of the material $\mathrm{Ni} 42 \mathrm{CrTi}$

From previous study in Section 5 , it can be deduced that for ring resonators made of alloy, it is impossible to eliminate frequency split only by promoting the geometric accuracy. This conclusion is helpful to design the machining accuracy of the resonator from a view of the manufacturing cost.

\section{Conclusions}

(1) Harmonic transformation of the parametric errors of the ring resonator is deduced. FEM results show that the frequency split caused by the $4^{\text {th }}$ harmonic error is much bigger than that caused by other harmonic error. The $4^{\text {th }}$ thickness harmonic error should be strictly controlled during the machining process.

(2) The roundness error of a typical ring resonator sample is measured and the harmonic transformation is implemented. Results show that for the tested resonator, its first 20 orders of harmonic waves contribute to the main geometric errors.

(3) An accurate FEM model with actual geometric errors is built. The simulation results show that the frequency split of the ring resonator is $0.6 \mathrm{~Hz}$. It shows that the geometric error only generates about $16.2 \%$ of the total frequency split. The other portion of the frequency split is caused by inhomogeneous material.

(4) A grouping experiment based on different alloys is designed. It can be observed under the same machining process, the frequency split of the copper resonator is smaller than that of Ni4iCrTi resonators. It means that for some metallic resonators, it is not possible to eliminate the frequency split only by promoting the geometric accuracy.

(5) The presented method in this paper provides useful information for the improvement of the resonator manufacture.

\section{Declaration}

\section{Acknowledgements}

The authors would like to thank to the Laboratory of Microsystems, National University of Defense Technology, China, for technical support and access to equipment.

\section{Funding}

Supported by National Natural Science Foundation of China (Grant No. 51935013)

\section{Availability of data and materials}

The datasets supporting the conclusions of this article are included within the article.

\section{Authors' contributions}

The author' contributions are as follows: $\mathrm{XX}$ was in charge of the whole trial and wrote the manuscript. All authors read and approved the final manuscript.

\section{Competing interests}

The authors declare no competing financial interests.

\section{Consent for publication}

Not applicable

\section{Ethics approval and consent to participate}

Not applicable

\section{References}

[1] V V Chikovani, I M Okon, A S Barabashov, et al. A set of high accuracy low cost metallic resonator CVG. Proceedings of the Position, Location and Navigation Symposium, Monterey, CA, May 5-8, 2008: 238-243.

[2] J Beitia, C Fell, I Okon, et al. Low cost CVG for high-grade north finders and targeting systems. Inertial Sensors \& Systems 
Symposium, Karlsruhe, Germany, Sep 16-17, 2014: 1-15.

[3] Zhinan Q, Tianliang Q, Yao P, et al. Optical and electrical method characterizing the dynamic behavior of the fused silica cylindrical resonator. Sensorss, 2019, 19, 2928.

[4] V V Chikovani, H V Tsiruk. Shock resistance of differential type ring-like resonator vibratory gyroscope. Electronics \& Control Systems, 2014, 4(42): 79-83.

[5] Y Wang, M H Asadian, A M Shkel. Compensation of frequency split by directional lapping in fused quartz micro wineglass resonators. Journal of Micromechanics \& Microengineering, 2018, 28, 095001 .

[6] J H Kim, J H Kim. Trimming of imperfect hemispherical shell including point mass distributions. International Journal of Mechanical Sciences, 2017, 131(132): 847-852.

[7] Y C Seong, H K Ji. Natural frequency split estimation for inextensional vibration of imperfect hemispherical shell. Journal of Sound and Vibration, 2011, 330(9): 2094-2106.

[8] C H J Fox. A simple theory for the analysis and correction of frequency splitting in slightly imperfect rings. Journal of Sound and Vibration, 1990, 142(2): 227-243.

[9] Y Wang, M H Asadian, A M Shkel. Modeling the effect of imperfections in glassblown micro-wineglass fused quartz resonators. Journal of Vibration and Acoustics, 2017, 139, 040909.

[10]B Paolo, C Giovanni. Frequency split and vibration localization in imperfect rings. Journal of Sound and Vibration, 2007, 306(35): 691-711.

[11]D Senkal, M J Ahamed, A A Trusov, et al. Achieving sub-Hz frequency symmetry in micro-glassblown wineglass resonators. Journal of Microelectromechanical Systems, 2014, 23(1): 30-38.

[12]M Weinberg. Symmetric gyroscope frequency separation by geometry and roughness. International Symposium on Inertial Sensors \& Systems, Laguna Beach, CA, Feb 25-26, 2014: 1-4.

[13]Y Tao, X Z Wu, D B Xiao, et al. Precision balance method for cupped wave gyro based on cup-bottom trimming. Chinese Journal of Mechanical Engineering, 2012, 26(1): 63-70.

[14]X Xi, X Z Wu, Y L Wu, et al. Modeling and analysis of mechanical Quality factor of the resonator for cylinder vibratory gyroscope. Chinese Journal of Mechanical Engineering, 2017, 30(1): 180-189.

[15]X Xi, X Z Wu, Y M Zhang, et al. A study on Q factor of the trimmed resonator for vibratory cupped gyroscopes. Sensors and Actuators A: Physical, 2014, 218(1): 23-32.

[16]Y M Zhang, X Z Wu, Y L Wu, et al. Nodal vibration and pattern angle error analysis of the imperfect resonators for vibratory cylinder gyroscopes. International Journal of Precision Engineering and Manufacturing, 2016, 17(4): 419-426.

[17]Y Yang, Z Fang, W Y Tan, et al. Balancing method for thin-walled resonator of low cost metallic vibratory gyroscope. Journal of Chinese Inertial Technology, 2013, 21(5): 672-675.

[18]Z Lin, M Y Fu, Z H Deng, et al. Frequency split elimination method for a solid-state vibratory angular rate gyro with an imperfect axisymmetric-shell resonator. Sensors, 2015, 15(2): 3204-3223.

[19]Y W Hu, Y P Zhou, H M Zhong, et al. Precise dynamic mass-stiffness balancing of cylindrical shell vibrating gyroscope along working modal axis. IEEE Sensors Journal, 2019, 19(22):

[24]V Matveev, M Basarab. Solid State Wave Gyro. Beijing: National Defense Industry Press, 2009. (in Chinese)

[25]Y M Zhang, Y L Wu, X Z Wu, et al. A Novel Vibration Mode Testing Method for Cylindrical Resonators Based on Microphones. Sensors, 2015, 15: 1954-1963.
10347-10354.

[20]W Gu, J B Tan. Research on the roundness error separation method based on single transposition characteristic angle. Journal of Optoelectronics Laser, 2015, 12(26): 112-116.

[21]R Viitalaa, T Widmaiera, B Hemmingb, et al. Uncertainty analysis of phase and amplitude of harmonic components of bearing inner ring four-point roundness measurement. Precision Engineering, 2018, 54: 118-130.

[22]G Augustin, G Ingrid. Application of Fourier Series for Evaluation of Roundness Profiles in Metrology. Advances in Science and Technology Research Journal, 2019, 13(4): 30-38.

[23]D Lynch. Coriolis vibratory gyros. Symposium Gyro Technology, Stuttgart, Germany, Sept 25-26, 1998.

\section{Biographical notes}

Xiang Xi, born in 1986, is currently an associate professor at College of Intelligence Science, National University of Defense Technology, China. He received his $\mathrm{PhD}$ degree from National University of Defense Technology, China, in 2014. His research interests include inertial sensors, micro-electromechanical systems and mechanical engineering.

Tel: +86-0731-84574958; E-mail: xixiang@nudt.deu.cn

Yong-Meng Zhang, born in 1988, is currently a lecturer at College of Intelligence Science, National University of Defense Technology, China. He received his $\mathrm{PhD}$ degree from National University of Defense Technology, China, in 2017. His research interests include inertial sensors, micro-electromechanical systems and control of gyroscope.

E-mail: zhangym@nudt.edu.cn

Jiang-Kun Sun, born in 1992, is currently a $\mathrm{PhD}$ candidate at College of Intelligence Science, National University of Defense Technology, China.

E-mail: sunjiangkun15@nudt.edu.cn

Xue-Zhong Wu, born in 1965, is currently a professor at National University of Defense Technology, China. He received his $\mathrm{PhD}$ degree from National University of Defense Technology, China, in 1988. His research interests include inertial sensors, micro-electromechanical systems and precise manufacture.

E-mail: ylwu_nudt@sina.com

\section{Appendix}

Appendix and supplement both mean material added at the end of a book. An appendix gives useful additional information, but even without it the rest of the book is complete: In the appendix are forty detailed charts. A supplement, bound in the book or published separately, is given for comparison, as an enhancement, to provide corrections, to present later information, and the like: A yearly supplement is issue. 
Figures

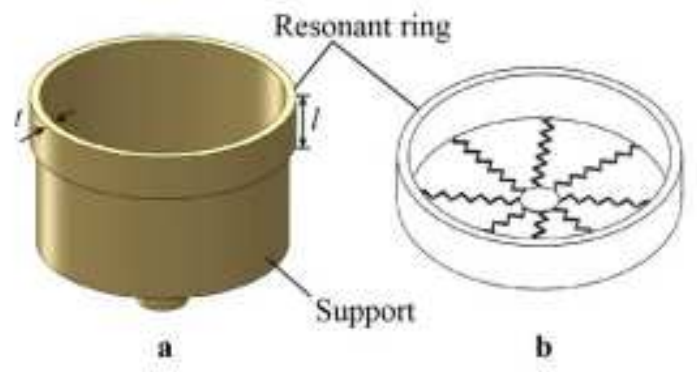

\section{Figure 1}

The metallic resonator and its ring model, a resonator, $b$ ring model

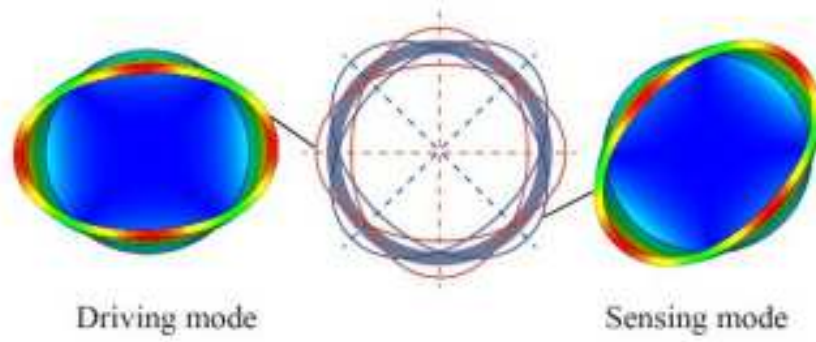

\section{Figure 2}

Operating mode of the ring resonator

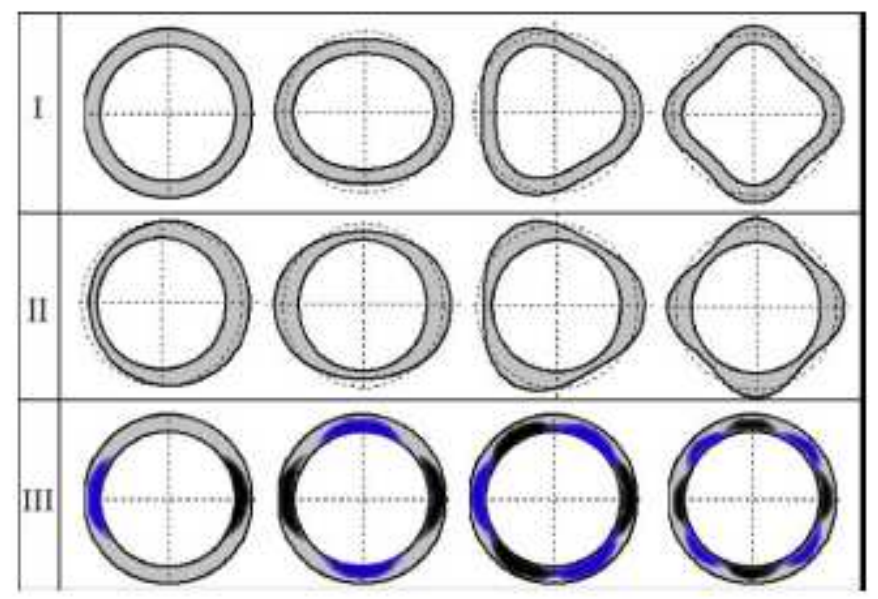

Figure 3

Decomposition of the geometric errors of the ring resonators 


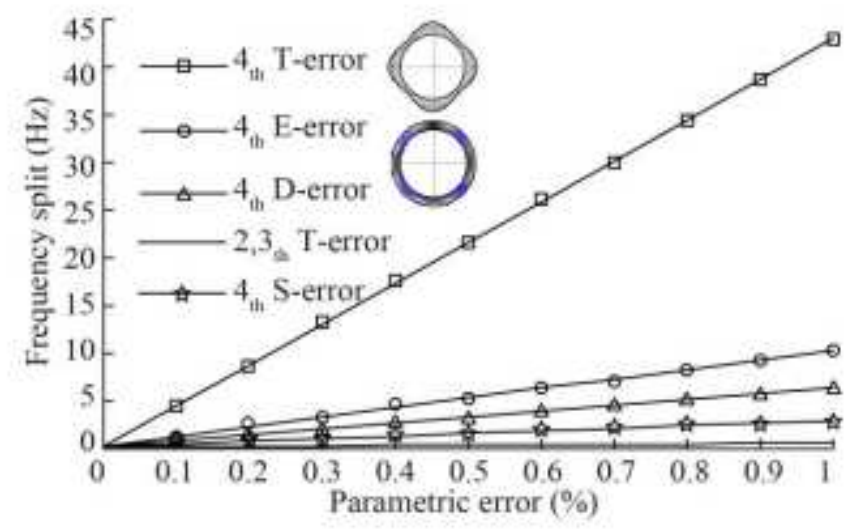

Figure 4

Frequency split dependent on harmonic wave order

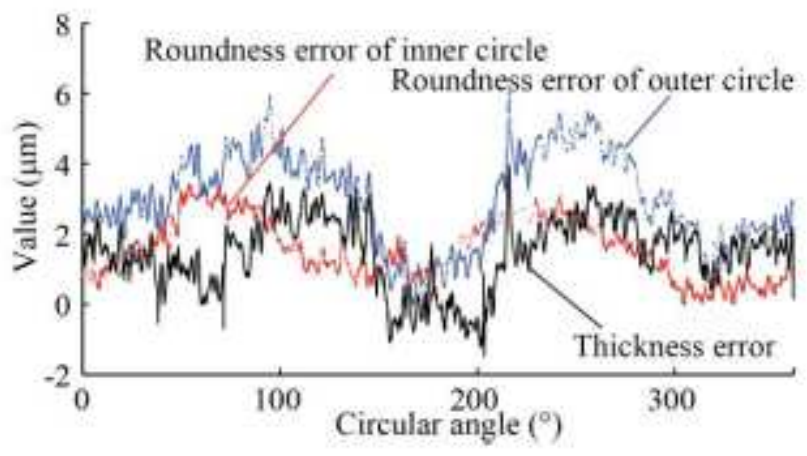

Figure 5

Roundness and thickness errors of the resonator.

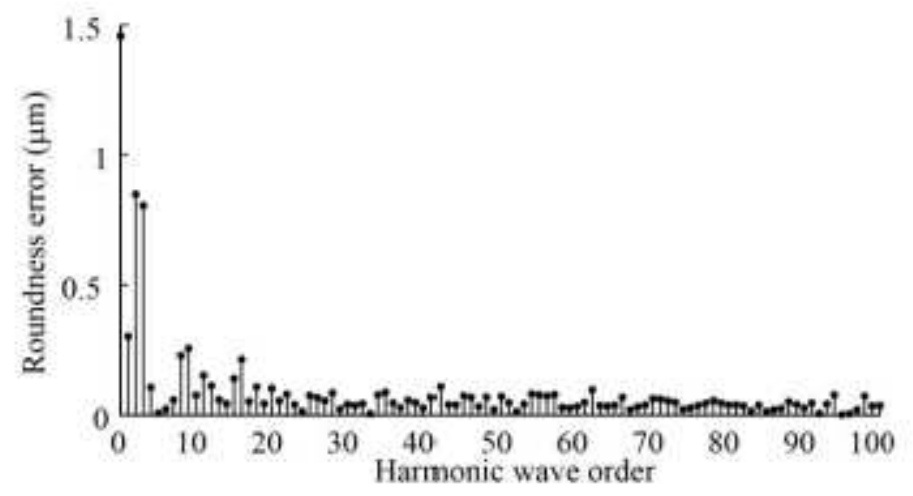

Figure 6

Roundness errors related to the orders of the harmonic waves 


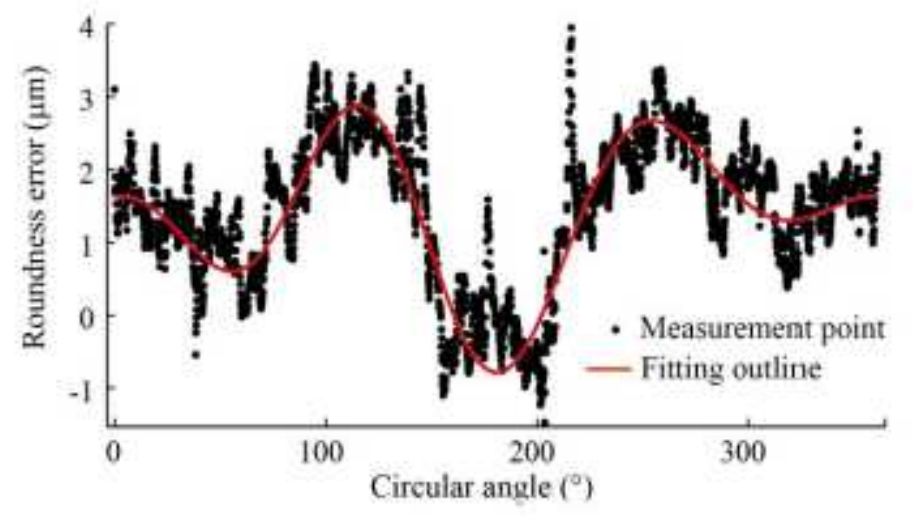

Figure 7

Fitting of roundness error using first 4 orders of harmonic waves

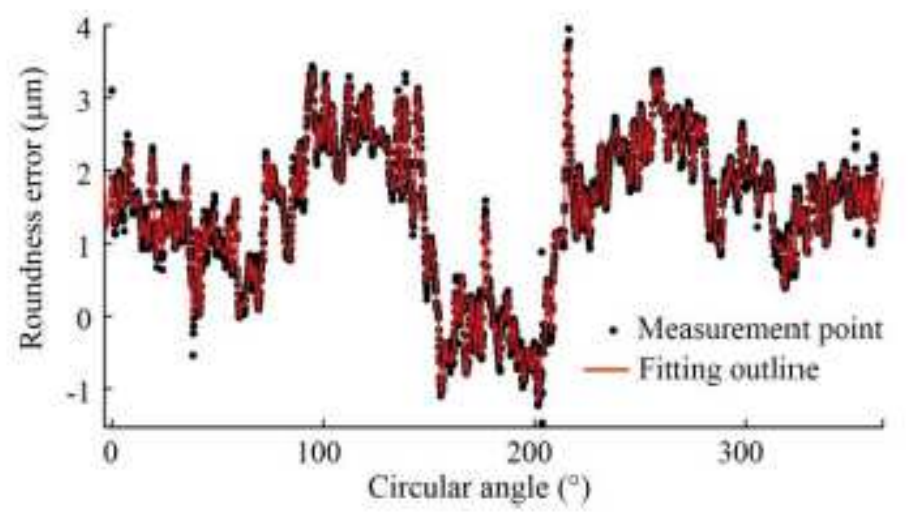

Figure 8

Fitting of roundness error using first 100 orders of harmonic waves

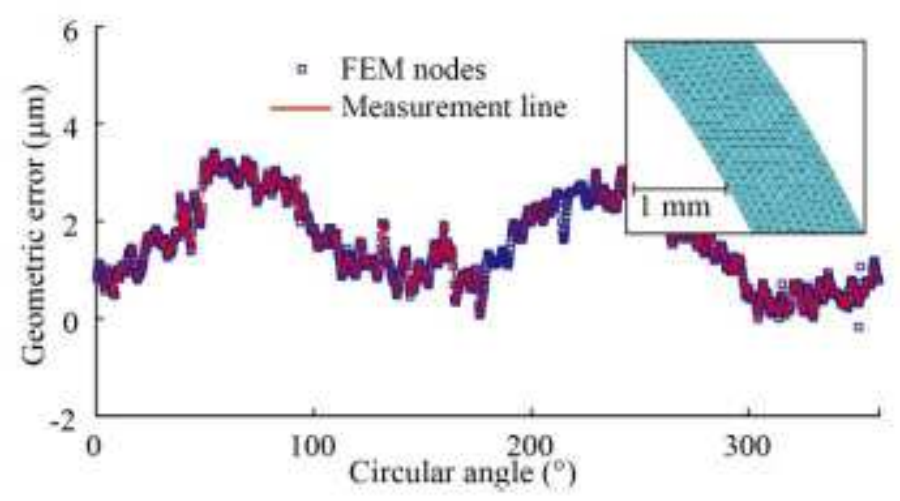

Figure 9

FEM modeling for frequency split analysis 


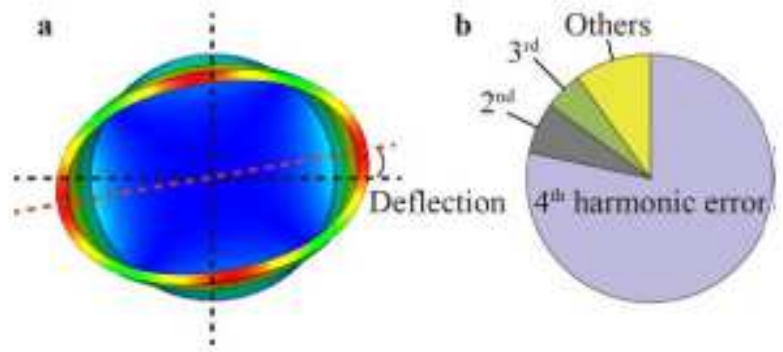

Figure 10

FEM result of the resonator, a eigenmode contour, $b$ frequency split dependent on different harmonic errors

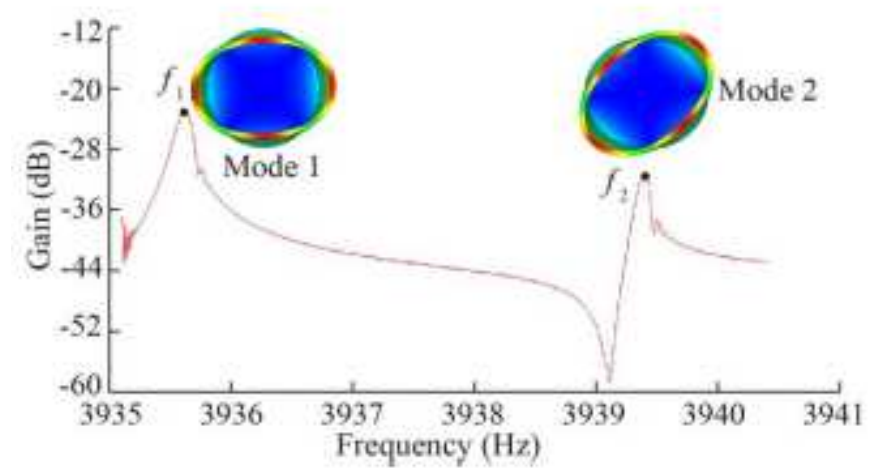

\section{Figure 11}

Frequency response of the imperfect ring resonator

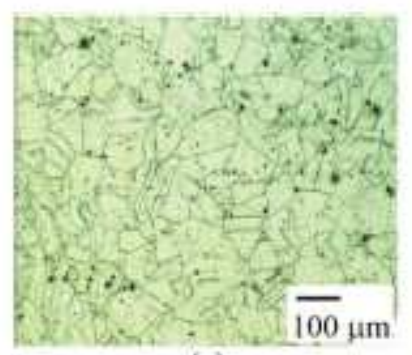

(a)

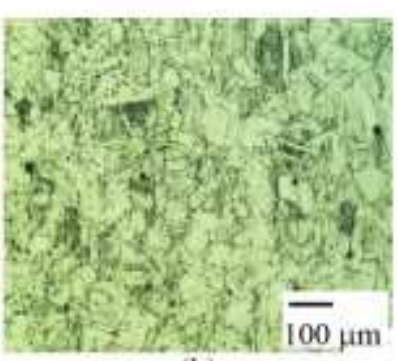

(b)

\section{Figure 12}

Metallograph from different positions of the alloy Ni42CrTi 


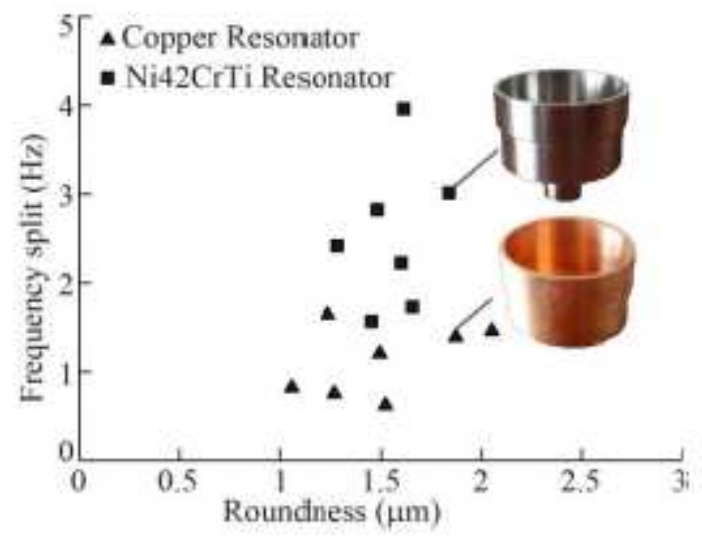

Figure 13

Metallograph from different positions of the material Ni42CrTi 